\title{
Immediate Resolution of a Grade 3 Varicocele Post-Prostatic Artery Embolisation (PAE)
}

\author{
Felice D’Antuono ${ }^{1}$ (D) Pietro Venetucci ${ }^{1}$
}

Received: 28 September 2017 / Accepted: 17 November 2017/Published online: 27 November 2017

(C) Springer Science+Business Media, LLC, part of Springer Nature and the Cardiovascular and Interventional Radiological Society of Europe (CIRSE) 2017

To the Editor,

We read with great interest the recent article by Tapping et al. [1]. The authors described the immediate resolution of a grade 3 left-sided male varicocele post-prostatic artery embolisation (PAE), suggesting that embolisation of the variable prostatic arterial branches may affect not only the arterial flow and end organ but also venous flow of other pelvic organs.

We compliment the authors for the paper; however, we would like to elaborate on the proposed theory for the resolution of the varicocele.

Firstly, the peri-prostatic venous plexus congestion and resulting increased flow to the medium and posterior pampiniform plexus should cause a secondary bilateral varicocele (due to the transcrotal venous anastomoses) rather than a monolateral one; also, the not sufficient capacity of the pudendal venous system should also cause haemorrhoids (through inferior rectal veins). We suggest an unacknowledged anatomical variant or a subtle pathological cause (i.e. right peri-prostatic plexus thrombosis) to explain a just left-sided varicocele.

Felice D'Antuono

felixdant@hotmail.it

1 Department of Advanced Biomedical Sciences, University of Naples "Federico II", Naples, Italy
Secondly, although a prostate with benign prostatic hyperplasia can be considerably increased by volume, it is hard to believe that this causes a pelvic arterial flow increase such as to entirely justify a grade 3 varicocele; furthermore, the complete resolution of the anterior pampiniform plexus (APP) ectasia in a clinically significant varicocele in $<24 \mathrm{~h}$ is very uncommon with retrograde sclerotherapy technique, in which APP appears immediately flow-free but still increased in size for several weeks due to inflammatory reaction, and we are interested in knowing what the authors mean by resolution (just plexus decompression or also its thrombosis).

\section{Compliance with Ethical Standards}

Conflict of interest Dr. Felice D'Antuono and Dr. Pietro Venetucci have no conflict of interest.

\section{Reference}

1. Tapping CR, Little MW, Boardman P. Immediate resolution of a grade 3 varicocele post prostatic artery embolisation (PAE). Cardiovasc Intervent Radiol. 2017;40(9):1481-3. 